\title{
A Study on the Web-based Distributed Design Application in the Preliminary Ship Design
}

\author{
Chang-Kyu Park, Member, KIMICS
}

\begin{abstract}
Today's engineering design is carried out in a distributed fashion geographically or physically. This places new requirements on the computational environments such as efficient integration and collaboration. With the advances of the Internet and Network environment recently, many researches have been proposed and at the same time, Web-based distributed design gives to a new paradigm in design and manufacturing fields. That is, Web-based technologies lead to reduce the product development times and to ensure a competitive product in order to exchange and interact of real-time design information that integrates the distributed design environment between departments as well as companies via Internet and Web. So, an efficient data communication for design information sharing is the foundation for collaborative systems in the distributed environment. Design data communication techniques such as CORBA, DCOM and RMI have been considered in the existing research but they have some problems that are limitations of interoperability and firewall problems in the Web. Therefore, this paper presents a Web-based distributed design application where distributed design information resources are integrated and exchanged using Web Services for supporting XML and HTTP without the interoperability and firewall problems through the $330 \mathrm{~K}$ VLCC case.
\end{abstract}

Index Terms - Integration, Collaboration, Web-based distributed design, Web Services

\section{INTRODUCTION}

Recently, due to the rapid advances of Internet and Network environment, many researches on the development of web based application programs are actively carried out in a various area, and thus new paradigm's change is appeared in the design and production areas for adopting web based distributed design concept.

In other words, by recognizing the limits of traditional sequential and independent design process, new design process using Internet and Web as an alternative is

Manuscript received August 8, 2010; revised September 6, 2010; accepted September 9, 2010.

Chang-Kyu Park is with the Department of Ship and Ocean, VISION University of Jeonju, Jeonbuk, 560-760, Korea (Email: ckpark@ jvision.ac.kr) suggested to develop a competitive product and to reduce a product development time through real time exchange of product design information by integrating a design environment between the companies or departments.

Besides, MDO (Multidisciplinary Design Optimization) method had developed as a systematic and an optimal design automation tool by considering multidiscipline related with product simultaneously.

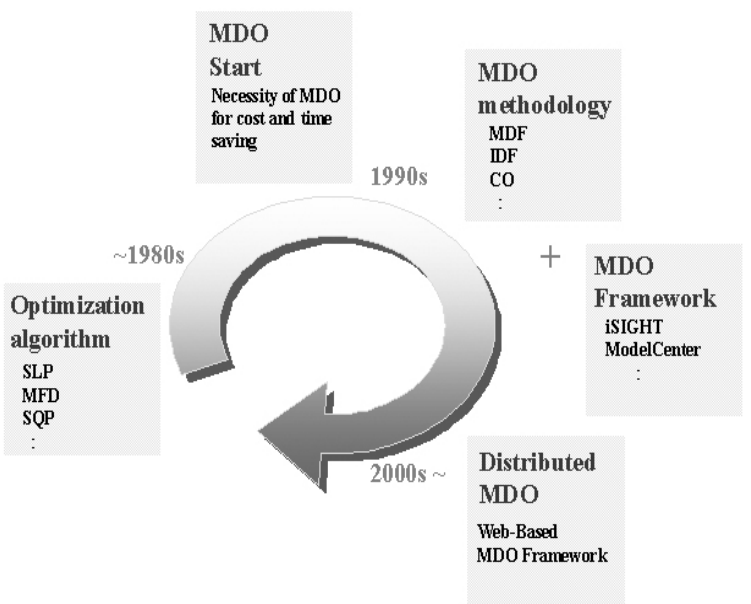

Fig. 1. The change and prospects of MDO[1].

In fact, as you can see in Fig. 1, around 1980, an optimization tool was developed for single objective case and sequential optimization problem cases. Since 1980 , more effective design methodology is required to design a complex engineering system. Thus, at early of 1990, new design framework research called MDO had started, and finished at the end of 1990 in the aero industry. However, due to IT web based MDO framework system will be required in the near future to effectively manage the various resources in the web environment.

Thus, the primary purpose of this research is set to the development of web based distributed design system by integrating MDO methodology and CAD \& CAE tools, where MDO is used for effective design method for complex system such as marine, aero and mechanical areas, and $\mathrm{CAD} \& \mathrm{CAE}$ for efficient design information supplier during design process [Fig. 2]. 


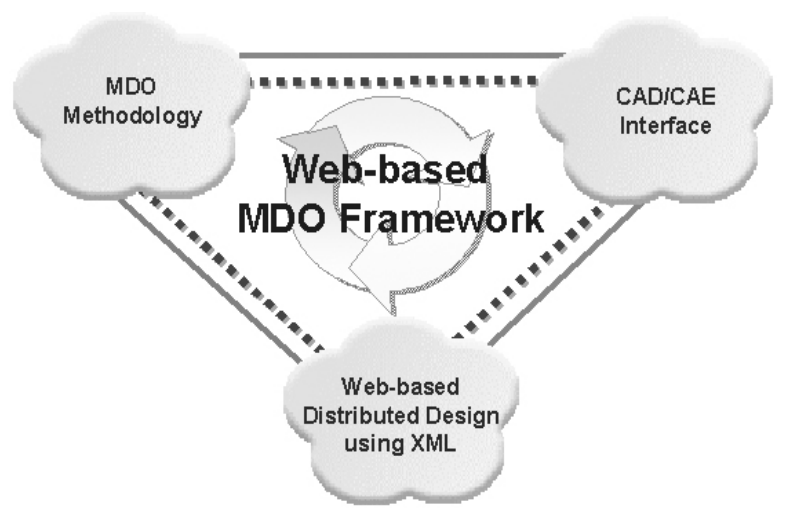

Fig. 2. The change and prospects of MDO.

\section{THE RELATED TECHNOLOGY STATUS}

For traditional distributed design, CORBA (Common Object Request Broker Architecture)[2], DCOM (Distributed Component Object Model)[3], RMI (Remote Method Invocation)[4] of JAVA were used as a communication tool.

However, these tools are compatible to the Intranet environment due to the limitation of interoperability and firewall problem between different platforms [Fig. 3]. Thus, to integrate the distributed various platforms as well as different languages effectively and to collaborate design tasks between departments within a company as well as between different companies, it might be necessary for distributed design method to have Web Services [6] using standard protocol of Internet such as HTTP (Hyper Text Transfer Protocol) and XML (eXtensible Mark-up Language)[5].

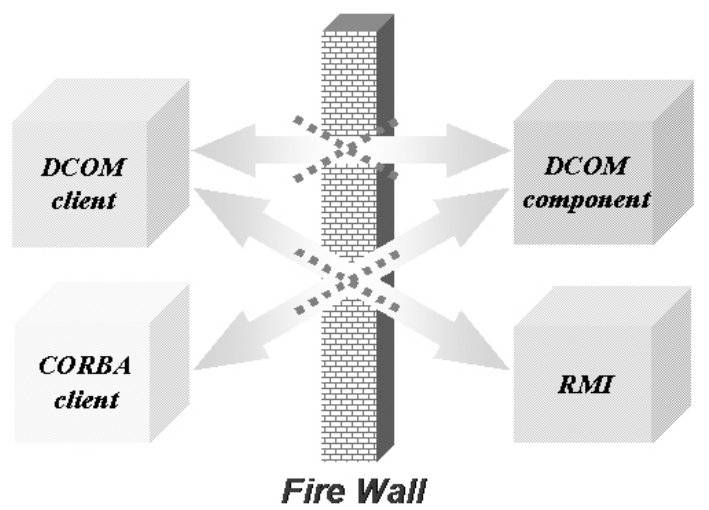

Fig. 3. The Problem of existing distributed design.

A brief concept of RPC (Remote Procedure Call) [7] as a basic tool of distributed design method is described first, and then various distributed design methods are discussed. The concept of RPC had been used long before the concept of Web. RPC is usually used to call the necessary program existed at different computer system through the network to obtain the necessary information for calculation like calling subprogram within the same computer system. Thus, the process when calling other program located at different system stops temporarily and waits until other called system returns the requested results to the original calling system. That is why we called this process of two systems Synchronous process.

\subsection{CORBA}

The CORBA (Common Object Request Broker Architecture) is an open distributed object-computing infrastructure being standardized by the OMG (Object Management Group). CORBA automates many common network programming tasks such as object registration, location, and activation; request demultiplexing; framing and error-handling; parameter marshalling and demarshalling; and operation dispatching.

CORBA provides a platform-independent, languageindependent architecture for writing distributed, objectoriented applications.

\subsection{DCOM}

The DCOM (Distributed Component Object Model) is a protocol that enables software components to communicate directly over a network in a reliable, secure, and efficient manner. DCOM is designed for use across multiple network transports, including Internet protocols such as HTTP. DCOM is based on the Open Software Foundation's DCE-RPC spec and will work with both Java applets and ActiveX components through its use of the COM (Component Object Model). Follow the links below to learn more about DCOM. DCOM extends the COM to support communication among objects on different computerson a LAN (local area network), a WAN (wide area network), or even the Internet.

\subsection{JAVA RMI}

Java RMI (Java Remote Method Invocation) enables the programmer to create distributed Java technologybased to Java technology-based applications, in which the methods of remote Java objects can be invoked from other Java virtual machines, possibly on different hosts. RMI uses object serialization to marshal and unmarshal parameters and does not truncate types, supporting true object-oriented polymorphism.

\subsection{SOAP}

SOAP is a lightweight protocol for exchange of information in a decentralized, distributed environment. It is an XML based protocol that consists of three parts:

SOAP envelope: an overall framework for expressing what is in a message, which should deal with it, and whether it is optional or mandatory.

SOAP encoding rules: a serialization mechanism that can be used to exchange instances of application-defined data types. 
SOAP RPC: defines a convention that can be used to represent remote procedure calls and responses.

SOAP is firmly rooted in the object-oriented paradigm. SOAP passes live objects, each with their own state information, across the Internet. This opens up a whole new range of possible applications.

Created by Userland, IBM, Developmentor, Lotus, and Microsoft, SOAP has been in development for a long time. Now SOAP is supported by IBM, Microsoft, and SUN, as well as a number of other companies.

TABLE I

\section{COMPARISONS OF DISTRIBUTED COMPONENT TECHNIQUES}

\begin{tabular}{|c|c|c|c|c|c|}
\hline $\begin{array}{c}\text { RPC } \\
\text { Probcol }\end{array}$ & CORBA & $\begin{array}{c}\text { DCOM } \\
\text { DSCHA }\end{array}$ & $\begin{array}{c}\text { Java RMI } \\
\stackrel{\text { Java }}{=}\end{array}$ & $\begin{array}{l}\text { SOAP } \\
\text { SOHPE }\end{array}$ & $\begin{array}{l}\text { Web Services } \\
\text { Neb Services }\end{array}$ \\
\hline Development & $\begin{array}{c}\text { OMG } \\
\text { (Object } \\
\text { Management } \\
\text { Group) }\end{array}$ & Mic ros oft & $\begin{array}{c}\text { Sun } \\
\text { Microsystems }\end{array}$ & $\begin{array}{l}\text { Microsoft } \\
\text { IBM }\end{array}$ & $\begin{array}{c}\text { W3C } \\
\text { (Recommended) }\end{array}$ \\
\hline Advantage & $\begin{array}{l}\text { Platform- } \\
\text { independent and } \\
\text { Language- } \\
\text { independent }\end{array}$ & $\begin{array}{l}\text { Language- } \\
\text { independent }\end{array}$ & $\begin{array}{l}\text { Platform- } \\
\text { independent }\end{array}$ & $\begin{array}{l}\text { - Platform- } \\
\text { independent and } \\
\text { Language } \\
\text { independent } \\
\text { - No firewall } \\
\text { problem }\end{array}$ & $\begin{array}{l}\text { - Platform- } \\
\text { independent and } \\
\text { Language- } \\
\text { independent } \\
\text { - No firewall } \\
\text { problem }\end{array}$ \\
\hline Disadventage & Firewall problem & $\begin{array}{l}\text { - Operated in MS } \\
\text { Windows Only } \\
\text { - Firewall problem }\end{array}$ & $\begin{array}{l}\text { Comm unications are } \\
\text { only possible Java } \\
\text { to Java }\end{array}$ & & \\
\hline
\end{tabular}

\subsection{Web Services}

Web services are the basis of distributed computing across the Internet. A Web service consumer locates a Web service and invokes the operations it provides. Web services use standard Web protocols such as XML, SOAP, and HTTP[8],[9]. Emerging Web services standards such as SOAP, Web Service Description Language (WSDL) and Universal Description, Discovery and Integration (UDDI) will enable system-to-system integration that is easier than ever before.

\section{IMPLEMENTATION}

Rapid progress of WWW affects engineering design environment as well as Business fields to utilize Web service technology to enhance it's competitively in the world. It is thus explained in the previous chapter that data transfer problems raised in the distributed design environment when using DCOM, CORBA, \& RMI could be avoid by adopting new Web service design methods using XML based standards data type and Hypertext Transfer Protocol, HTTP. In the present example we had chosen, using .NET [10] system developed by MS is applied to demonstrate the possibility and usefulness of Web service application to the geographically distributed design process.
In this paper, we had shown two examples of Web based Framework. Its basic Client/Server system is shown in Fig. 4.

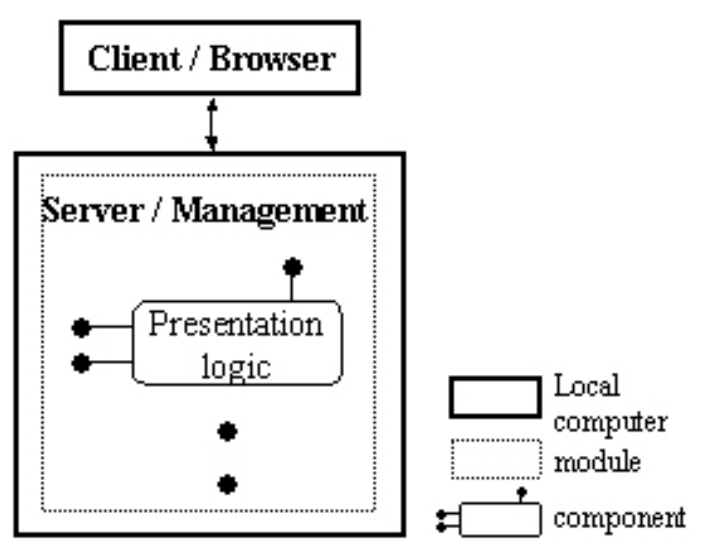

Fig. 4. Client/Server system architecture.

Client application is taken as 'Thin client' case that is ease to publish and manage.

Server side is constructed by two or three modulebased systems, which could be used to other system. Each module in local computers might have several components.

The system architecture of I-Beam design example case is organized by three modules and Preliminary Ship Design example is only one module, which has several components.

\subsection{I-Beam Design Example}

Simple beam design problem shown in Fig. 5 is chosen to show the applicability of web service for structural optimization problem that is often appeared in the traditional structural optimal design cases by using FEM code such as ANSYS. Here, Application Program Interface (API) calls ANSYS program as well as optimization program during the optimization process automatically to find the minimum weight optimum design.
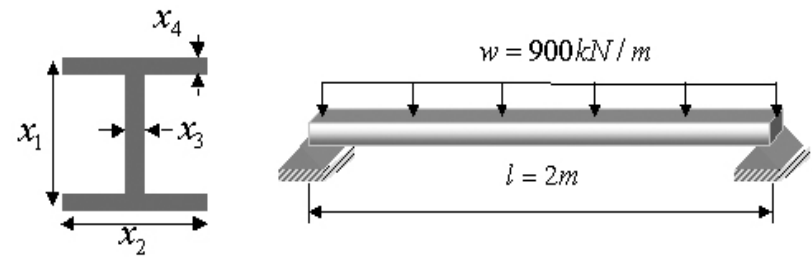

Fig. 5. I-beam optimal design.

Following is an optimization problem formulation for I-beam design case.

Minimize Area, $A=2 x_{x} x_{4}+x_{3}\left(x_{1}-2 x_{4}\right)$ 
Find.

$$
x_{1}, x_{2}, x_{3}, x_{4}
$$

\section{Subject to.}

$$
\begin{aligned}
g_{1}(x)= & \sigma-\sigma_{a} \leq 0 ; \text { Stress } \\
& g_{2}(x)=\delta-\delta_{a} \leq 0 ; \text { Displacement }
\end{aligned}
$$

Where,

$$
\begin{array}{ll}
0.1 \leq x_{1} \leq 0.8 & 0.1 \leq x_{2} \leq 0.8 \\
0.001 \leq x_{3} \leq 0.05 & 0.001 \leq x_{4} \leq 0.05
\end{array}
$$

Fig.6 shows a Web-based OPT Framework used for this case, where Server A \& B for Ansys program and another server for optimization process are constructed.

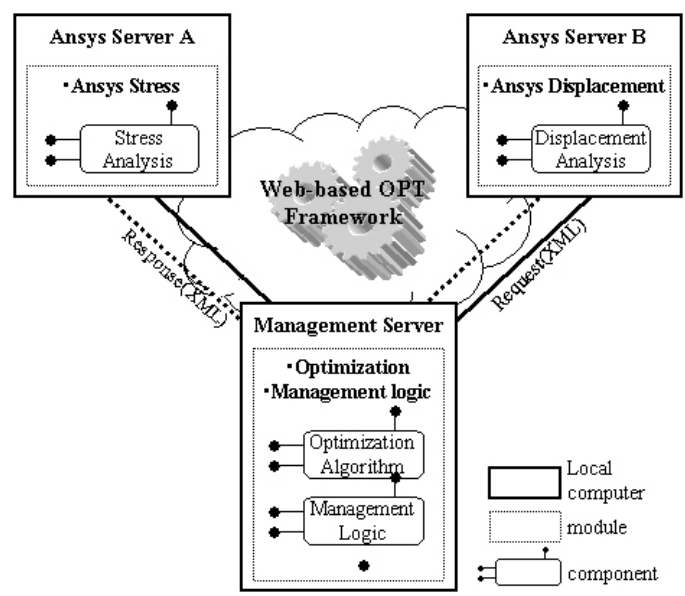

Fig. 6. Web-based OPT framework.

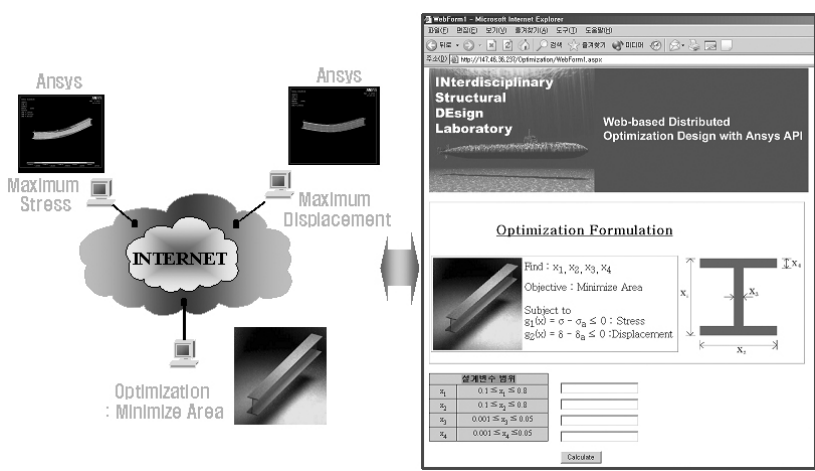

Fig. 7. I-beam design problem using Web Services.

Final implemented web service is schematically shown in Fig. 7.

To solve I-Beam Design Problem, first of all, ANSYS Server A \& B are assigned to handle Max. Stress Constraint \& Max. Displacement respectively. Though these two constraints are handled together by any ANSYS
Server, we are assumed that these two constraints are obtained from different disciplines such as structural analysis \& vibration problem at the moment.

Of course, each server is activated by Web service using XML \& HTTP for document \& transfer protocol to obtain the necessary information such as Max. stress \& Max. displacement. After that, optimization task is allocated to another Server, which calls and brings the necessary data from other servers to solve an optimization problem. Then, each server can give or take a necessary data from each other. If data transfer occurs between servers, then it looks like P2P (Peer-To-Peer) case.

Thus, for multi clients to use this framework anywhere, any place, and any time whenever If Internet were available, this would be another merit of Web service, compared with traditional framework.

Assuming data communication between the servers operates smoothly, Client could solve I-Beam design problem effectively by following process after connecting Internet.

(1) Open Internet Explorer on Client's Windows

(2)Input URL (Uniform Resource Location) for an appropriate server address (http://147.46.36.237/ Optimization/WebForm1.aspx) at the Internet Address bar where optimization task of I-Beam Design Problem is assumed to be solved properly.

(3) If new window is open as shown in Fig. 7, input an initial design value $\left(x_{1}, x_{2}, x_{3}, x_{4}\right)$ of design parameters, which are already programmed in this optimization server. (4) Click Calculation Button appeared in the lower place of Internet Explorer

(5) Once input such an initial value, Server A \& server B at remote place calculate it's necessary information and send its value to optimization server. This process will iterate until final min. weight of beam cross section is obtained. Thus, when converged at optimal solution, server displays final objective value to Web Browser used by Client.

\subsection{Preliminary Ship Design Example}

This example is to determine the principal dimensions of ship during the preliminary ship design of 330K DWT VLCC [11], and final optimization problem can be formulated as follow.

Given: DWT (Deadweight), CV (Cargo Volume), T (Design draft), V (Ship speed)

Find (Design Variables: L (Length), B (Breadth), D (Depth), $C_{B}$ (Block coefficient), $D_{P}$ (Propeller diameter), $P_{i}$ (Propeller pitch), $A_{E} / A_{o}$ (Propeller blade area ratio)

Minimize Building Cost

Subject to

$g_{1}:$ Buoyancy-Weight equilibrium

$g_{2}:$ Minimum required freeboard condition

$g_{3}:$ Required cargo volume constraints 
$g_{4}:$ Initial stability condition

$g_{5}$ : Obesity coefficient conditions for maneuvering

$g_{6}:$ Recommended block coefficient by Watson \&

Gilfillan

$g_{7}$ : Propeller must absorb a torque transmitted from the main engine

$g_{8}$ : Propeller must deliver a thrust required by the ship in certain speed

$g_{9}$ : Propeller must deliver a thrust required by the ship in certain speed

For this preliminary ship design case, Web service is constructed by using only one server to handle the principal dimension of ship since there is no need to call any special CAE tools for ship design problem, in contrast to the previous case, where one server connected by client call ANSYS tools remotely through Web browser. Of course, various algorithms needed for calculating resistance, stability, freeboard, and etc. are installed in the server as shown in Fig. 8.

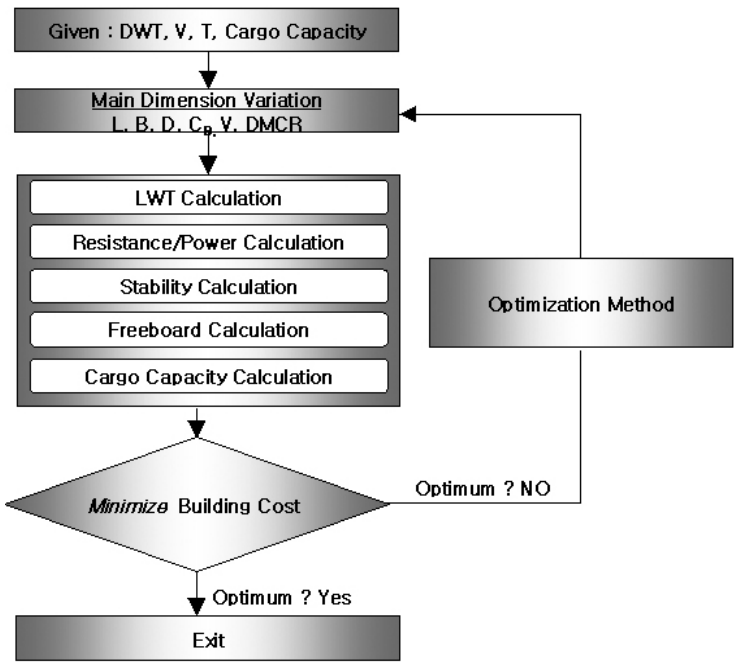

Fig. 8. Preliminary ship design problem algorithm.

(1) As usual, Open Internet Explorer on Client's Window.

(2) Input URL (uniform resource location) corresponding an appropriate server address (http://147.46.36.237/ Ship_Client/WebForm1.aspx) at the address bar where optimization task of preliminary ship design Problem is programmed properly.

Thus, final Web service results are delivered to client in the Web browser by using XML and HTTP. In this case, after connecting Internet by client, if following procedure will be executed, then final solution will be obtained easily.
(3) If new window is open as shown in Fig. 9, input an initial design value $\left(L, B, D, C_{B}, V, D M C R\right)$ of ship parameters.

(4) Click Calculation Button appeared in the lower place of Internet Explorer.

(5) Once inputted such an initial value, server calculates build cost, and checks whether it is optimal or not. If not, it will iterate until final min. building cost is obtained.

Thus, when converged, a final objective value will be shown on Web Browser.

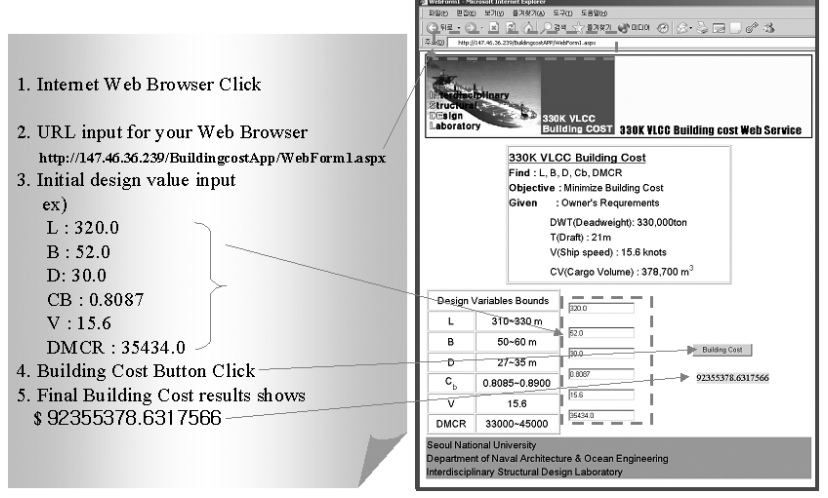

Fig. 9. 330K VLCC example using Web Services.

\section{CONCLUSIONS}

Nowadays, geographically or physically distributed design teams basically do engineering design. Thus, it is necessary for the design team to be supported by new computing environment by which collaboration and integration of different design tasks can be established easily. As a result, distributed design method is essential for the integration process of various resources, but following problems have to be considered properly due to the heterogeneity of systems.

- Geographically distributed designer's attitude

- Physically distributed characteristics of data, languages, and platforms.

○ Different Communication protocol

To resolve the above problems appeared in the engineering areas, it is essential for computer system to have the following architecture.

- Standardized Data Structure: XML

- Standardized Communication Protocol for distributed data: HTTP

o Platform independence

- Developed language interoperability

$\circ$ Firewall problem for security 
Web service is well known distributed design tool suggested by W3C (World Wide Web Consortium) using HTTP (Hyper Text Transfer Protocol), XML (eXtensible Markup Language), SOAP (Simple Object Access Protocol) and WSDL (Web Service Description Language). Since it uses Industry standard technology, Platform dependency, language dependency problems as well as firewall problem could be avoid so that a distributed design could be managed without much difficulties, compared with convention network system.

Therefore, in this study, we had investigated Web based distributed design method using .NET for I-Beam design as well as preliminary ship design cases, and found that there are much possibilities of using Web service for geographically distributed engineering design environment.

\section{REFERENCES}

[1] Y.S, Yang, C. K. Park and W. S. Ruy, "Integrated Design System using MDO and Approximation Technique", Computational Structural Engineering Institute of Korea, pp.275-283, 2004(in Korea).

[2] CORBA Spec., http://www.corba.org

[3] DCOM, www.microsoft.com/tech/DCOM.asp

[4] JAVA RMI, http://java.sun.com/products/jdk/rmi

[5] XML 1.0, W3C, 1998, http://www.w3.org/TR/REC-XML

[6] Web Services, http://www.w3.org/2002/ws/

[7] S. Laurent, J. Johnson, and E. Dumbill, "Programming Web Services with XML-RPC", O'Reilly, 2001.

[8] SOAP Spec, http://www.w3.org/TR/SOAP

[9] SOAP1.2Spec, http://www.w3.org/TR/SOAP12

[10] Visual Studio .NET, http://msdn.microsoft.com/vstudio/

[11] Y.S, Yang, C. K. Park and W. S. Ruy, "A Study on the Preliminary Ship Design Method using Deterministic Approach and Probabilistic Approach", Journal of SNAK, Vol.41, No.3, pp.49-59, June 2004(in Korean)

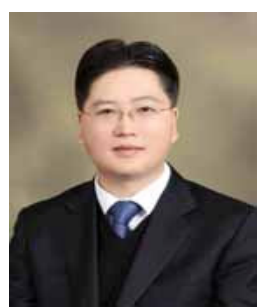

Chang-Kyu Park received the $\mathrm{Ph}$. D. degrees in Department of Naval Architecture and Ocean Engineering from Soul National University, Seoul, Korea, in 2007. From March, 2009, he has been the faculty of Department of Ship and Ocean, VISION University of Jeonju, Jeonbuk, Korea. His research interests include Web-based Network, CAD/ CAE and Structural Design. COSEIK, KOSMEE, KOSME, KSOE and KSME. 